Research Article

\title{
Experimental Study on Quasi-Static Pressure of Slot Hydraulic Blasting
}

\author{
Chengwei Liu $\mathbb{D}^{1,2}$ Binwei Xia $\mathbb{D}^{1,2}{ }^{1,2}$ Yiyu Lu $\mathbb{D}^{1,2}$ and Yugang Gao $\mathbb{D}^{1,2}$ \\ ${ }^{1}$ State Key Laboratory of Coal Mine Disaster Dynamics and Control, Chongqing University, Chongqing 400044, China \\ ${ }^{2}$ College of Resources and Environmental Science, Chongqing University, Chongqing 400030, China \\ Correspondence should be addressed to Binwei Xia; xbwei33@cqu.edu.cn
}

Received 4 September 2018; Accepted 27 November 2018; Published 16 December 2018

Academic Editor: Fiorenzo A. Fazzolari

Copyright ( $\odot 2018$ Chengwei Liu et al. This is an open access article distributed under the Creative Commons Attribution License, which permits unrestricted use, distribution, and reproduction in any medium, provided the original work is properly cited.

Air is commonly used as the noncoupling borehole medium in directional slot blasting where the quasi-static pressure is small, the energy utilization rate of the explosive is low, and the breaking efficiency of the coal-rock mass is not high. This study investigates the effect of quasi-static pressure on crack propagation in closed-field blasting. Crack length propagation in the quasi-static pressure stage accounts for more than $60 \%$ of the total crack length. Water is therefore proposed as the noncoupling medium of the slotting borehole to increase the quasi-static pressure. A series of experiments was performed to investigate and compare quasi-static pressures generated using noncoupled water-medium blasting and noncoupled air-medium blasting. The experimental results show that the quasi-static pressure is 37-46 times larger in water-medium blasting than in the air-medium case. The experimental measurements show good agreement with theoretical analysis. The results show that the energy utilization rate of the explosives in the hydraulic slotting blasting is high with a notable energy storage effect, and that the fracturing range can be significantly increased. Additionally, fracture by directional blasting using this approach can be more controllable.

\section{Introduction}

Blasting is a widely used method for rock mass crushing in mining. The power source of rock fracturing during blasting is mainly divided into shock wave and quasi-static pressure. The rock mass breaking mechanism under shock waves has been widely studied while quasi-static pressure, which is much smaller in scale, has received less attention. Rock breakage in practical blasting results jointly from both sources [1]. After the blasting wave passes quickly through the rock mass and the impact has generated the initial fracture and field damage, the continuous expansion of the subsequent crack is powered mainly by the static pressure of the detonation gas [2-4].

Quasi static pressure in explosive building demolition was studied by Feldgun et al. [5], and Liu et al. and Zhang et al. studied quasi-static pressure characteristics in the explosions [6, 7]. Quasi-Static pressures in these studies were generated under noncoupled air conditions, in which air was used as the transmission medium for the blasting energy between the explosive and borehole walls. However, because of the compressibility of air, the quasi-static pressure formed by its expansion is low and the fracture propagation range is limited. Li and Ma, Huang et al., Zhang et al. studied the characteristics of hydraulic blasting $[1,8,9]$, which involves high-energy transmissions. The formation of a "water wedge" effect and bubble pulsation phenomenon is also conducive to the breakage of coal-rock mass and continuous expansion of fractures. However, these features have not yet been discussed in terms of static pressure. At present, only Zong and Xuan have theoretically discussed the quasi-static pressure of hydraulic blasting [10].

Replacement of the filling media among the explosives and the borehole, however, requires noncoupled charging conditions. Directional slot fracturing methods, which have been used in the precise excavation of roadways [11, 12], low damage mining [13], directional roof fracturing to eliminate mine pressure [14], and directional coal fracturing to increase permeability [15], effectively applies the required noncoupled conditions. The present study therefore proposes the application of slotting filled by a water medium. The quasi static pressure characteristics of water are studied 
experimentally, analysed theoretically, and compared with the quasi-static pressure of air. Theoretical guidance for the construction of Slot Hydraulic Blasting is also provided.

\section{Materials and Methods}

To study the role of quasi-static pressure in the blasting process, closed-field blasting experiments were carried out on plexiglass specimens in three borehole shapes: (1) circular; (2) slotting; and (3) square. The airtightness of the specimens is shown in Figure 1. A charge of lead azide was used with a $100 \mathrm{mg}$ dosage installed in the hole of each test specimen to achieve explosive charging. The crack propagation process was recorded by a high-speed photographic system consisting of a digital camera (Photron, Fastcam-SA5 (16G), Japan) and a solid-state laser (Figure 2).

Observation of the blasting process demonstrated that the shock wave generated by blasting propagates rapidly from the borehole to the periphery. Only the initial cracking was generated by this action, and the crack itself did not expand forward following the shock wave. When the explosion process reached $90 \mu \mathrm{s}$, the shock wave had spread beyond the field of view, and the specimen was no longer affected by the shock wave action. Subsequent detonation gas was clearly visible at this time, and the crack continued to expand under the quasi-static pressure formed by the detonation gas until the crack volume increased. Crack propagation ceased when the pressure was reduced below the specimen fracturing level. As such, the stages of crack propagation were identified, and the extended lengths were counted (Figure 3). The specimens in the three different boreholes all showed that, under airtight conditions, the crack length propagated in the quasi-static pressure stage accounted for more than $60 \%$ of the total crack length. In terms of blasting technology applied in mining, the boreholes are required to be blocked to form a closure. An increase of the quasi-static pressure is therefore important for improving the blasting effect.

2.1. Experiments. Experiments were performed to study the quasi-static pressure characteristics in slot hydraulic blasting using a slotting explosive chamber. Explosions under waternoncoupled charging and air-noncoupled charging conditions were investigated using three different doses. The characteristics of quasi-static water and quasi-static air pressure were measured and analysed. The experimental group design is listed in Table 1.

2.1.1. Explosive Containers and Explosives. The slotting explosive chamber consisted of upper and lower parts (Figure 4), and the container was fastened by bolts. A silicone film was placed at the interface to ensure sealing of the upper and lower interfaces. The tip angle of the slot was $60^{\circ}$, the radius was $45 \mathrm{~mm}$, the radius of the upper and lower planes was $28 \mathrm{~mm}$, and the seam height was $20 \mathrm{~mm}$. Explosives were made of gunpowder, packed in $6 \times 20 \mathrm{~mm}$ cylindrical chargings with doses of 150,200 , and $250 \mathrm{mg}$. Electronic ignition was used for detonation (Figure 5).

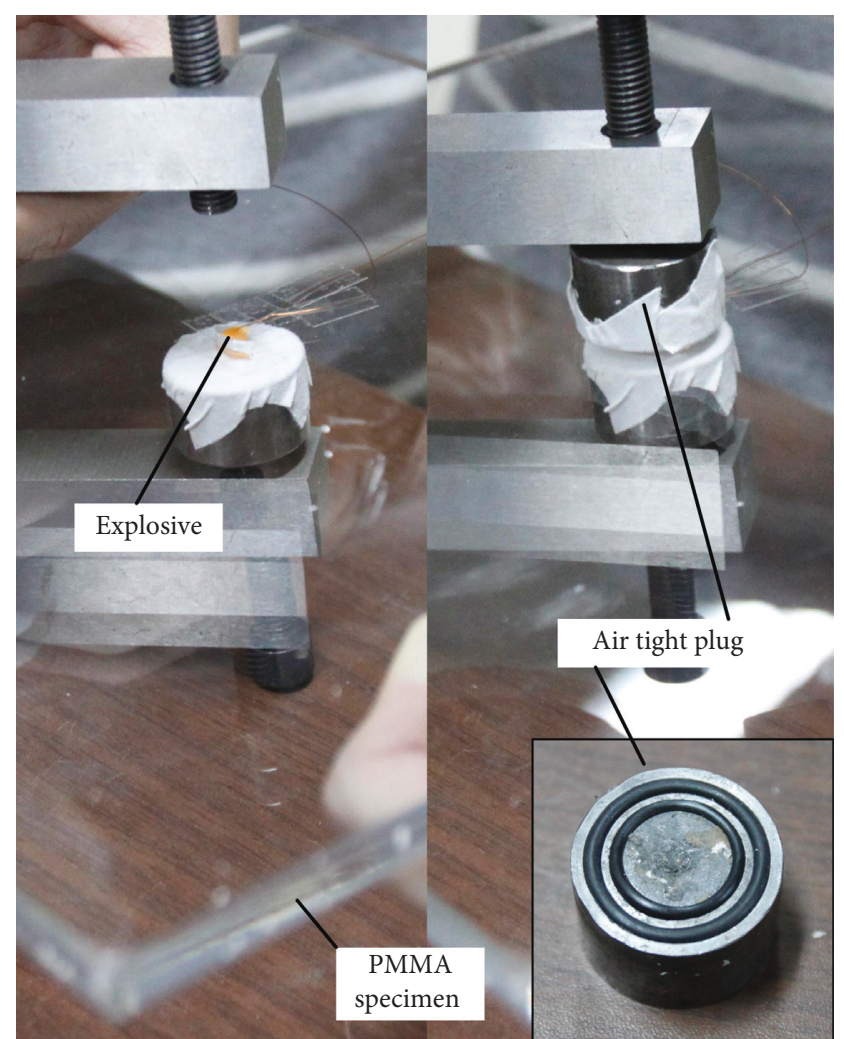

FIgURE 1: Airtightness of the specimens.

2.1.2. Equipment and Sensors. Data were acquired using a VIB-1274F (by Sichuan Tuopu Measurement and Control Technology Co., Ltd.) with a synchronously sampling 4channel analogue signal. The highest sampling rate per channel can simultaneously reach $10 \mathrm{Msps}$, as shown in Figure 6. During installation, the sensor was connected through an annular conduit to the detonation container to attenuate the shock wave and prevent the sensor from being damaged. The frequency response of the test was also reduced [16]. A piezoresistive sensor with a frequency response of $100 \mathrm{kHz}$ was used as the duration of the quasi-static pressure became longer and the pressure drop gentler, compared to the explosion shock wave. The $1 \mathrm{MPa}$ and $10 \mathrm{MPa}$ ranges were selected to measure the quasi-static pressure of the noncoupled air and noncoupled water charge blasting.

\section{Results}

Experiments were performed one time per group, and the pressure-time curves obtained in the explosive chamber are shown in Figure 7. Because of the presence of the annular conduit, the rises and falls of the impact pressure tended to be slow and the peak level was attenuated. Nevertheless, this design better meets the demands for testing the quasi-static pressure of an internal explosion field. The original curve displayed noise due to experimental interference. The original data were therefore taken $1: 1$; the experimental explosion pressure curve was obtained after the elimination of noise and the quasi-static pressure was measured through the curve, as shown in Figure 8. 

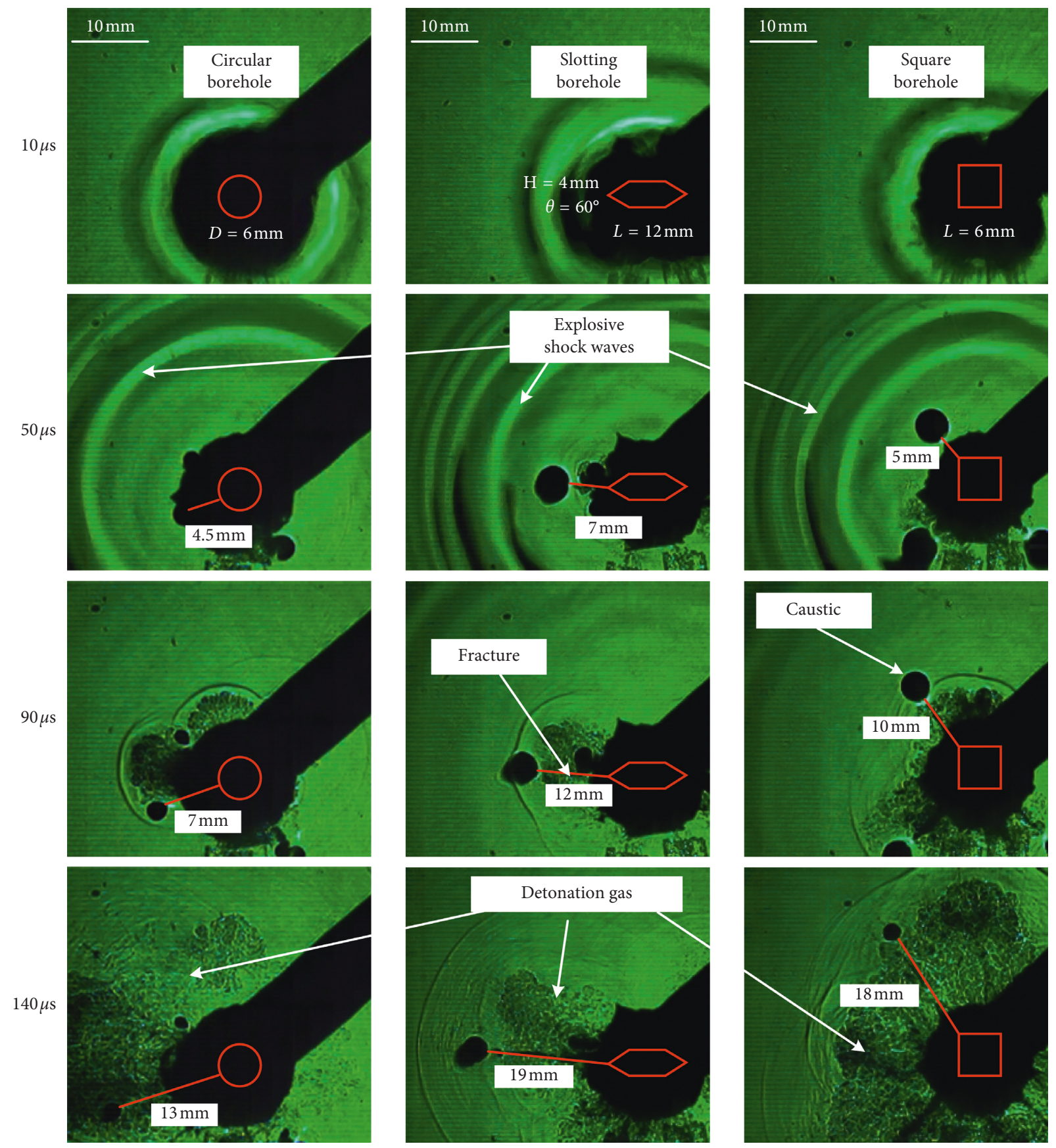

$\# 1$

\#2

\#3

FIgURe 2: Propagation process of the blasting fractures. Note: caustic is a dark spot formed by stress concentration at the crack tip in a dynamic caustics experiment, which is used to indicate the position of the crack tip in this figure.

Quasi-Static pressure was measured following the method recommended by Anderson et al. [17], that is, inversely drawing a new straight line at the nearly liner area of the curve, which leads the new line to intersect the rising curve at the $\mathrm{A} \sim \mathrm{F}$ point. Therefore, the ordinates of the point are the quasi-static pressure value. The quasi-static pressures of the six experimental groups are listed in Table 2.

The experimental data demonstrate that the quasi-static pressure during slotting detonation is consistently higher (37-46 times) using water as the coupling medium compared with air. The rate of energy utilization of the explosive is high when using water as the coupling borehole medium with a clear energy storage effect.

\section{Discussion}

After the shock wave generated by the explosion quickly passes through the borehole, the detonation product continues to expand and compress the original borehole medium. During this process, the newly added detonation gas 


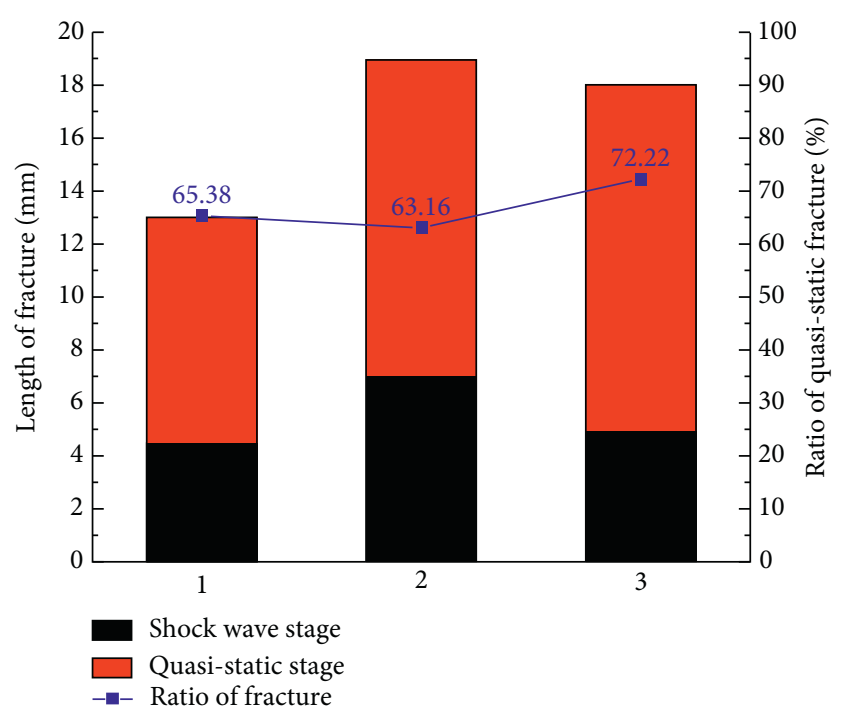

Figure 3: Fracture length at different stages.

TABLe 1: Design of experimental groups.

\begin{tabular}{lccc}
\hline Medium & \multicolumn{4}{c}{$\begin{array}{c}\text { Charge weight } \\
200 \mathrm{mg}\end{array}$} \\
\hline \multirow{4}{*}{$\begin{array}{lc}\text { Test groups } \\
\text { Air }\end{array}$} & $150 \mathrm{mg}$ & 2 & 3 \\
Water & 4 & 5 & 6 \\
\hline
\end{tabular}

expands and the pressure decreases. On the contrary, the original medium is compressed leading to an increase in pressure. The expansion and compression process ends when the new detonation product expands to a pressure equal to that of the original borehole medium after compression, at which time the equilibrium state is reached (i.e., pressures in the borehole are equal) and stress on the walls of the holes form quasi-static stress.

For simplicity, one can suppose that the explosion gas expansion follows the ideal equation of state, that is,

$$
P V^{\gamma}=\text { const, }
$$

where $P$ is the instantaneous absolute pressure during gas expansion and compression, $V$ is the volume of gas corresponding to $P$, and $\gamma$ is the adiabatic index, which is taken to be 1.3. After the explosion of the noncoupled air charge, the detonation gas pressure falls to the quasi-static equilibrium:

$$
P_{\mathrm{c}} r_{\mathrm{c}}^{2 \gamma}=P_{\mathrm{A}}\left(r_{\mathrm{c}}+b_{\mathrm{a}}\right)^{2 \gamma}
$$

where $P_{\mathrm{c}}$ is the gas pressure upon completion of the explosion, $r_{\mathrm{c}}$ is the charge radius equal to $3 \times 10^{-3} \mathrm{~m}, P_{\mathrm{A}}$ is the quasi-static pressure of the air at equilibrium, and $b_{\mathrm{a}}$ is the amount of radial expansion of the detonation gas, which is also the radial compression of the original gas in the borehole.

When the original gas pressure in the borehole rises to quasi-static equilibrium, it satisfies

$$
P_{0}\left(r_{0}-r_{\mathrm{c}}\right)^{2 \gamma}=P_{\mathrm{A}}\left(r_{0}-r_{\mathrm{c}}-b_{\mathrm{a}}\right)^{2 \gamma},
$$

where $P_{0}$ is the original gas pressure in the borehole, which is equal to atmospheric pressure $\left(1.01 \times 10^{5} \mathrm{~Pa}\right)$, and $r_{0}$ is the inner radius of the borehole and the equivalent inner radius of the slotting borehole $\left(3.65 \times 10^{-2} \mathrm{~m}\right)$. After the explosion of the noncoupled water charge, the detonation gas pressure falls to the quasi-static equilibrium, satisfying

$$
P_{\mathrm{c}} r_{\mathrm{c}}^{2 \gamma}=P_{\mathrm{w}}\left(r_{\mathrm{c}}+b_{\mathrm{w}}\right)^{2 \gamma}
$$

where $P_{\mathrm{w}}$ is the static pressure at equilibrium and $b_{\mathrm{w}}$ is the amount of radial expansion of the detonation gas, which is also the extent of radial compression of the water.

According to fluid mechanics theory, the relationship between $V$ and $P$ during water compression is

$$
d P=-\frac{E_{\mathrm{w}}}{V} d V
$$

Because $V=\pi\left(r_{0}^{2}-r^{2}\right) h$ and $d V=-2 \pi h r d r$, equation (5) becomes

$$
d P=-\frac{2 E_{\mathrm{w}} r}{r_{0}^{2}-r^{2}} d r
$$

where $E_{\mathrm{w}}$ is the bulk modulus of water, taken to be $2.1 \times$ $10^{9} \mathrm{~Pa}$. The following relation can be applied upon reaching equilibrium:

$$
\int_{P_{0}}^{P_{\mathrm{W}}} d P=\int_{r_{\mathrm{c}}}^{r_{\mathrm{c}}+b_{\mathrm{W}}} \frac{2 E_{\mathrm{w}} r}{r_{0}^{2}-r^{2}} d r,
$$

for which the integral is

$$
P_{\mathrm{w}}-P_{0}=E_{\mathrm{w}} \ln \left[\frac{r_{0}^{2}-r_{\mathrm{c}}^{2}}{r_{0}^{2}-\left(r_{\mathrm{c}}+b_{\mathrm{w}}\right)^{2}}\right] .
$$

Values obtained in the experiments were substituted into the theoretical derivation for calculation and verification. Since the explosive gas pressure at the time of explosion completion cannot be measured, $P_{\mathrm{A}}$ is substituted into equation (3). The insertion of $P_{\mathrm{A}}$ and calculated $b_{\mathrm{a}}$ into equation (2) allows determination of $P_{c}$. Equations (4) and (8) are then combined with the known parameters substituted therein. Finally, $b_{\mathrm{w}}$ and additional results are substituted into equations (4) or (8) to calculate $P_{\mathrm{w}}$.

Compare the theoretically derived calculation results with the experiment results, as shown in Table 3.

The theoretically derived values and experiment results are in good agreement (Table 3). Both datasets show that the energy utilization rate of the explosive is high when water is used as the borehole-coupling medium, with a clear energy storage effect. The detonation gas generated in the explosion expands and compresses the borehole medium, reaching equilibrium to form a quasi-static pressure. Because water is a microcompressive medium, the density only increases by about $5 \%$ when the external pressure increases to $108 \mathrm{~Pa}$. The pressure decrease of the detonation gas of the noncoupled air blasting is obvious under constant borehole volume, while that of noncoupled water blasting is slow. Hence, the quasi-static pressure of the noncoupled water is much greater than the noncoupled air counterpart at equilibrium. In practice, preexisting cracks often exist in the rock mass and the coefficient of moisture filtration of the air is greater than that of water. After the quasi-static pressure phase is formed, the gas in noncoupled air blasting is more likely to 


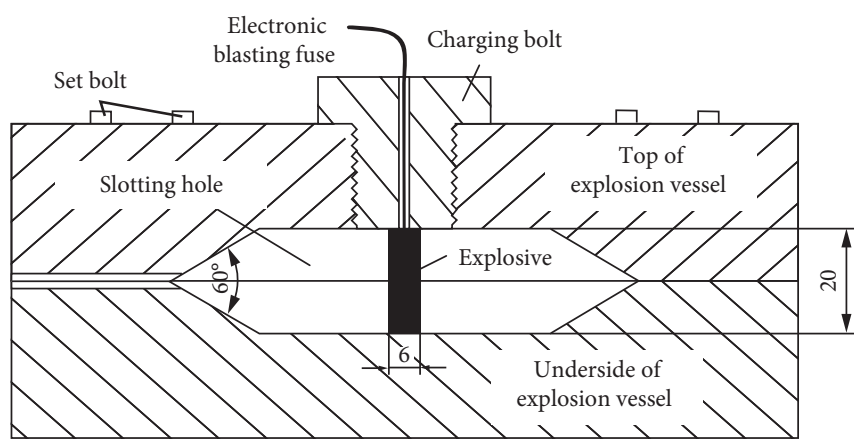

(a)
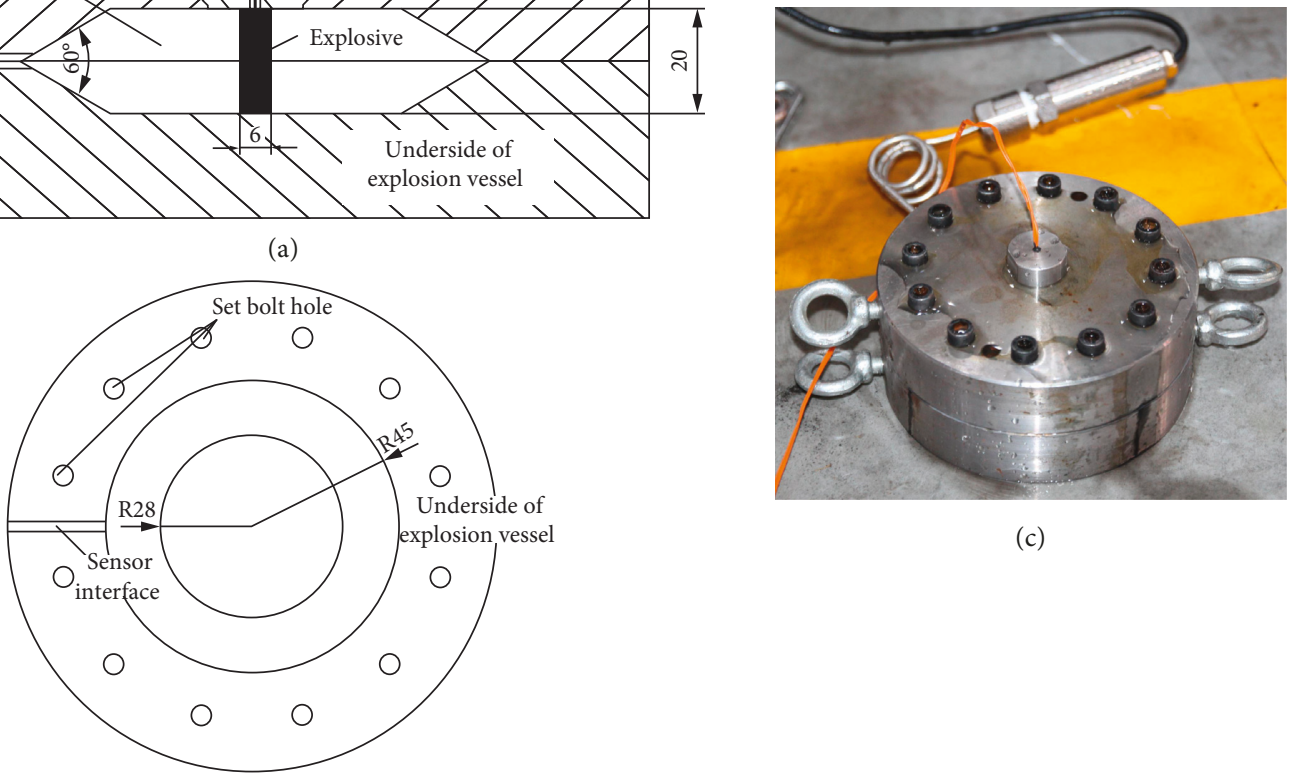

(c)

(b)

FIgURE 4: Slotting explosive chamber: (a) sectional view; (b) top view of underside; (c) photograph.

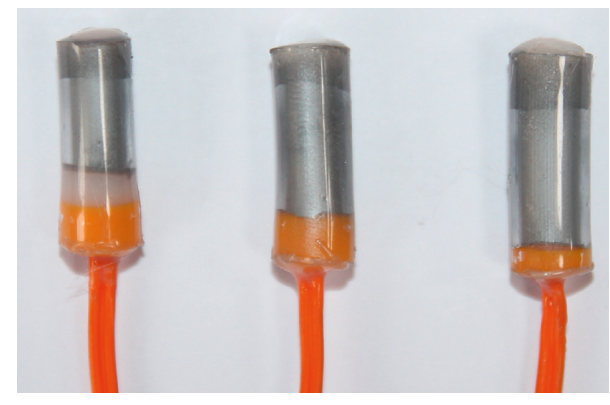

Figure 5: Photo of explosives.

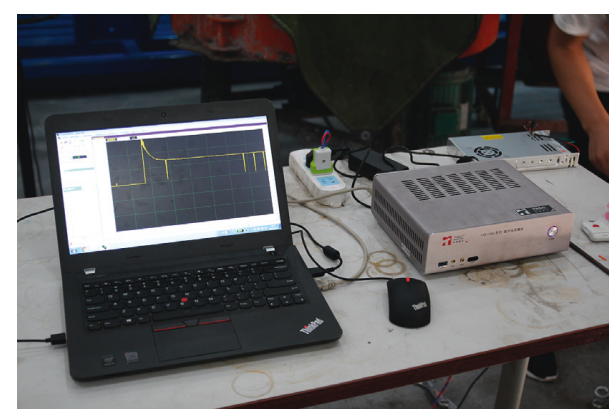

FiguRe 6: Data collection system.

filter moisture, forming a rapid pressure drop. The quasistatic pressure of coupled water blasting is not only larger than coupled air blasting in size, but also holds pressure longer. This is more conducive to the formation of a "water wedge effect," or wedging into the fracture tip, which promotes fracture propagation and larger breakage to achieve an improved blasting effect. Moreover, detonation gas expands slower in water than in air. As such, the pressure energy stresses are more even and gentle on the surrounding coalrock mass so that the coal-rock body produces cracks without plastic flow and excessive pulverization, which is more favourable for fracture controlling in directional blasting.

\section{Conclusions}

The effects on crack initiation and propagation in stress wave and quasi-static pressure stages were investigated experimentally. The results show that the quasi-static pressure stage provides the main power for fracture propagation in closed-space blasting. Crack length propagation during the quasi-static pressure stage accounts for more than $60 \%$ of the total crack length. An increase of the pressure magnitude and duration of the quasi-static pressure stage is therefore more conducive to increasing the length of the blast fracturing and improving the blasting effect in a variety of sealed blasting mining operations.

The use of water as the filling medium in a noncoupled borehole in directional blasting is proposed. Noncoupled air blasting and noncoupled water blasting experiments using equivalent boreholes with 150, 200, and $250 \mathrm{mg}$ chargings were performed in six groups to measure the respective quasi-static pressures. The experimental results show that 


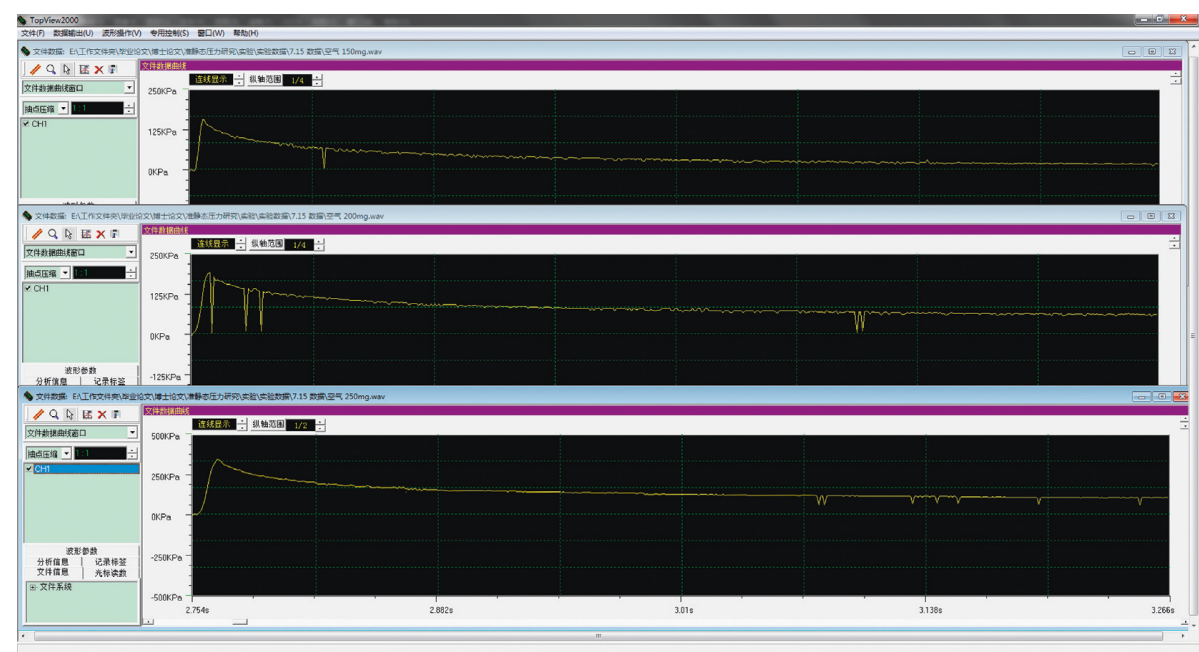

(a)

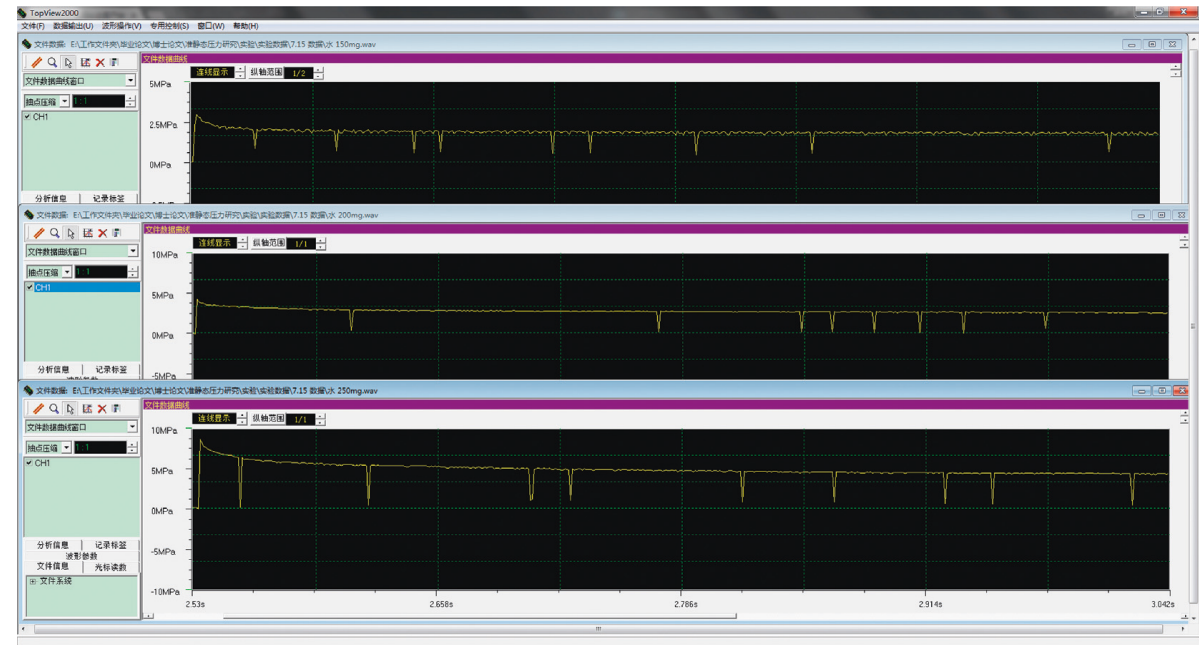

(b)

FIGURE 7: Explosion pressure and quasi-static pressure original curves from (a) noncoupled air charging and (b) noncoupled water charging.

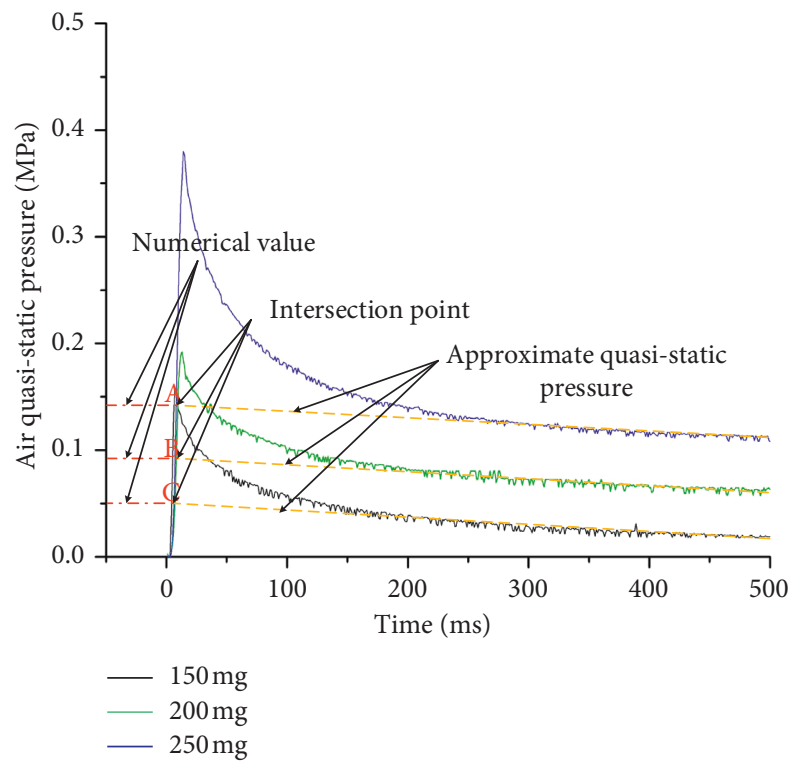

(a)

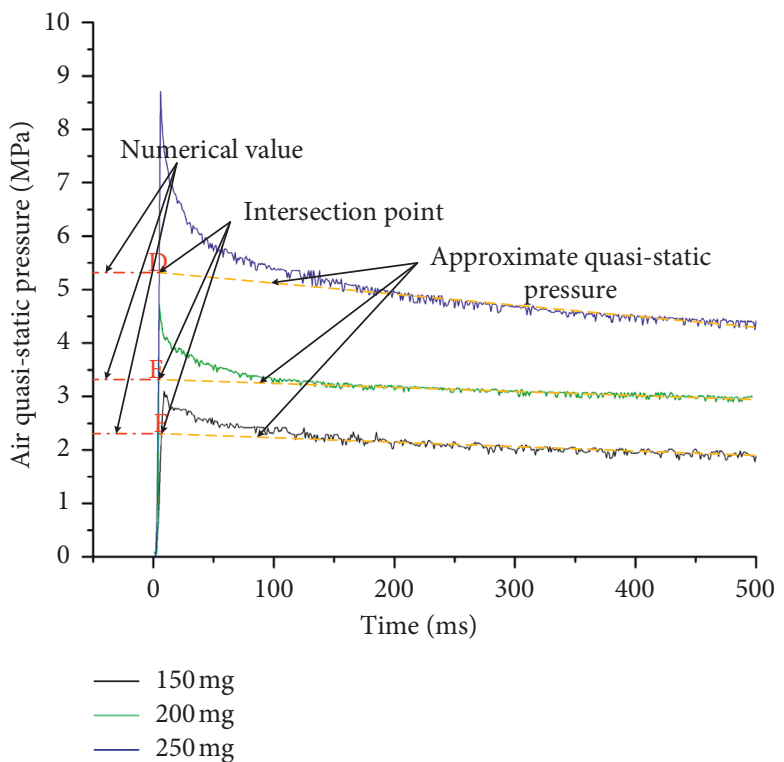

(b)

FiguRE 8: Method for measuring quasi-static pressure from (a) noncoupled air charging and (b) noncoupled water charging. 
TABLE 2: Experimental quasi-static pressures.

\begin{tabular}{lccc}
\hline Medium & \multicolumn{3}{c}{ Charge weight } \\
& $150 \mathrm{mg}$ & $200 \mathrm{mg}$ & $250 \mathrm{mg}$ \\
\hline Air $(\mathrm{Pa})$ & $5 \times 10^{4}$ & $9 \times 10^{4}$ & $1.4 \times 10^{5}$ \\
Water $(\mathrm{Pa})$ & $2.3 \times 10^{6}$ & $3.3 \times 10^{6}$ & $5.3 \times 10^{6}$ \\
\hline
\end{tabular}

TABLE 3: Theoretical calculation result.

\begin{tabular}{lccc}
\hline & $150 \mathrm{mg}$ & $200 \mathrm{mg}$ & $250 \mathrm{mg}$ \\
\hline$P_{\mathrm{Ag}}(\mathrm{Pa})$ & $5 \times 10^{4}$ & $9 \times 10^{4}$ & $1.4 \times 10^{5}$ \\
$P_{\mathrm{A}}(\mathrm{Pa})$ & $1.51 \times 10^{5}$ & $1.91 \times 10^{5}$ & $2.41 \times 10^{5}$ \\
$b_{\mathrm{a}}(\mathrm{m})$ & 0.0048 & 0.00728 & 0.00952 \\
$P_{\mathrm{c}}(\mathrm{Pa})$ & $1.81 \times 10^{6}$ & $4.7 \times 10^{6}$ & $9.9 \times 10^{6}$ \\
$b_{\mathrm{w}}(\mathrm{m})$ & 0.000161 & 0.00035 & 0.000592 \\
$P_{\mathrm{w}}(\mathrm{Pa})$ & $1.58 \times 10^{6}$ & $3.52 \times 10^{6}$ & $6.19 \times 10^{6}$ \\
$P_{\mathrm{wtg}}(\mathrm{Pa})$ & $1.48 \times 10^{6}$ & $3.42 \times 10^{6}$ & $6.09 \times 10^{6}$ \\
$P_{\text {wg }}(\mathrm{Pa})$ & $2.3 \times 10^{6}$ & $3.3 \times 10^{6}$ & $5.3 \times 10^{6}$ \\
Measured multiples & 46 & 36.67 & 37.86 \\
Theoretical multiples & 29.6 & 38.04 & 43.52 \\
\hline
\end{tabular}

Note: $P_{\mathrm{A}}$ and $P_{\mathrm{w}}$ are absolute pressures, $P_{\mathrm{Ag}}$ and $P_{\mathrm{wg}}$ are measured gauge pressures, and $P_{\mathrm{wtg}}$ is the theoretically calculated gauge pressure.

with different chargings, the quasi-static pressure of noncoupled water blasting is much larger (37-46 times) than that of noncoupled air blasting.

The quasi-static pressure formation process of noncoupled air blasting and noncoupled water blasting was analysed theoretically. The experimental values are consistent with theoretical calculations. Both datasets verify that use of a water medium enhances the quasi-static pressure stage. The reasons for noncoupled water blasting compared with noncoupled air blasting are discussed as well as its advantages in practice. The results show that when water is used as the noncoupling medium of the borehole, the energy utilization rate of the explosives is high, the energy storage effect is notable, and the blasting fracture range can be significantly extended with more controllable directional blasting. This study therefore provides theoretical support for the field application of slot hydraulic blasting.

\section{Data Availability}

All data included in this study are available upon request to the corresponding author.

\section{Conflicts of Interest}

The authors declare no conflicts of interest.

\section{Authors' Contributions}

Chengwei Liu, Binwei Xia, Yiyu Lu, and Yugang Gao conceived and designed the experiments, analysed the data, and wrote the paper. Chengwei Liu and Yugang Gao performed the experiments.

\section{Acknowledgments}

This work was jointly supported by the National Key Basic Research Program of China (No. 2014CB239206), the
National Natural Science Foundation of China (No. 51625401), and the Program for Changiiang Scholars and Innovative Research Team in University of China (No. IRT13043).

\section{References}

[1] Y. Q. Li and S. Z. Ma, Explosion Mechanics, Science Press, Beijing, China, 1992, in Chinese.

[2] A. S. Paine and C. P. Please, "An improved model of fracture propagation by gas during rock blasting-some analytical results," International Journal of Rock Mechanics and Mining Sciences \& Geomechanics Abstracts, vol. 31, no. 6, pp. 699-706, 1994.

[3] R. H. Nilson, W. J. Proffer, and R. E. Duff, "Modelling of gasdriven fractures induced by propellant combustion within a borehole," International Journal of Rock Mechanics and Mining Sciences \& Geomechanics Abstracts, vol. 22, no. 1, pp. 3-19, 1985.

[4] X. L. Yang and M. S. Wang, "Mechanism of rock crack growth under detonation gas loading," Explosion and Shock Waves, vol. 21, no. 2, pp. 111-116, 2001.

[5] V. R. Feldgun, Y. S. Karinski, I. Edri, and D. Z. Yankelevsky, "Prediction of the quasi-static pressure in confined and partially confined explosions and its application to blast response simulation of flexible structures," International Journal of Impact Engineering, vol. 90, pp. 46-60, 2016.

[6] W. X. Liu, D. Z. Zhang, F. P. Zhong et al., "Quasi-static pressure in a spherical explosion containment vessel," $E x$ plosion and Shock Waves, vol. 38, no. 5, pp. 1045-1050, 2018.

[7] Y. L. Zhang, R. Z. Li, H. Y. Jiang et al., "Experimental study of the characteristics of internal explosion pressure and power of thermobaric explosive," Acta Armamentarii, vol. 39, no. 07, pp. 1333-1338, 2018.

[8] B. Huang, C. Liu, J. Fu, and H. Guan, "Hydraulic fracturing after water pressure control blasting for increased fracturing," International Journal of Rock Mechanics and Mining Sciences, vol. 48, no. 6, pp. 976-983, 2011.

[9] Y. H. Zhang, W. Ni, G. C. Yin et al., "Study on improving the penetrability of coal seam with the water pressure blasting in the through beds hole," Journal of China Coal Society, vol. 03, pp. 298-302, 2004.

[10] Q. Zong and S. Q. Xuan, "Quasi static pressure of hydropressure blasting and its destruction judgement," West-China Exploration Engineering, vol. 9, no. 2, pp. 39-40, 1997.

[11] J.-G. Kim and J.-J. Song, "Abrasive water jet cutting methods for reducing blast-induced ground vibration in tunnel excavation," International Journal of Rock Mechanics and Mining Sciences, vol. 75, pp. 147-158, 2015.

[12] Y. Kang, D. F. Su, X. C. Wang et al., "Research on technology of light shock controlled blasting with water jet slotting," in Proceedings of 13th International ISRM Congress, Montréal, Canada, May 2015.

[13] M. P. Roy, P. K. Singh, M. Sarim, and L. S. Shekhawat, "Blast design and vibration control at an underground metal mine for the safety of surface structures," International Journal of Rock Mechanics and Mining Sciences, vol. 83, pp. 107-115, 2016.

[14] J. Yang, C. Liu, and B. Yu, "Application of confined blasting in water-filled deep holes to control strong rock pressure in hard rock mines," Energies, vol. 10, no. 11, p. 1874, 2017.

[15] B. W. Xia, C. W. Liu, Y. Y. Lu et al., "Experimental study of propagation of directional fracture with slotting hydraulic 
blasting," Journal of China Coal Society, vol. 41, no. 2, pp. 432-438, 2016.

[16] R. Z. Li, H. B. Zhai, X. M. Yan et al., "Test method research for the quasi-static pressure on inside-explosive," Chinese Journal of Sensors and Actuators, vol. 29, no. 2, pp. 208-212, 2016.

[17] C. E. Anderson, W. E. Baker, D. K. Wauters, and B. L. Morris, "Quasi-static pressure, duration, and impulse for explosions (e.g. HE) in structures," International Journal of Mechanical Sciences, vol. 25, no. 6, pp. 455-464, 1983. 


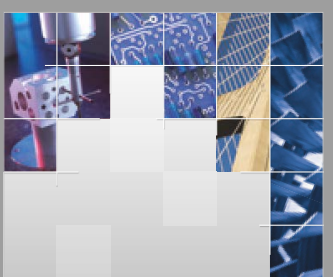

\section{Enfincering}
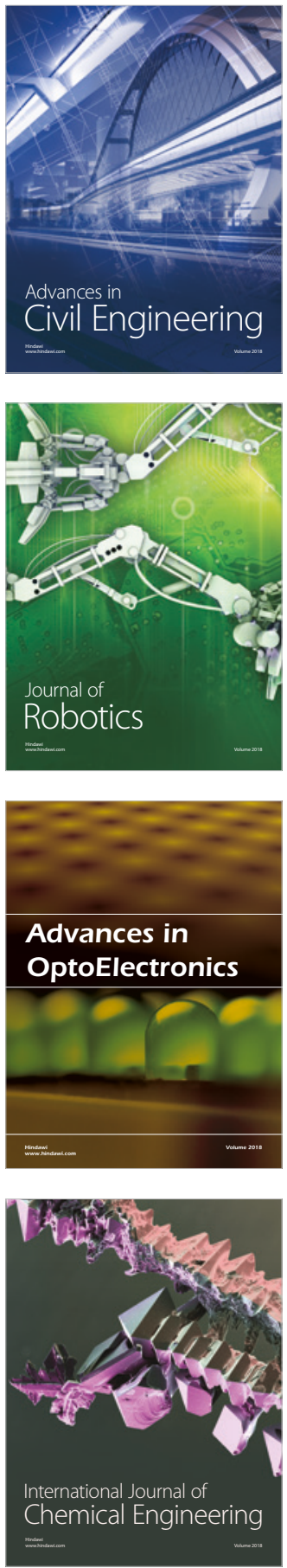

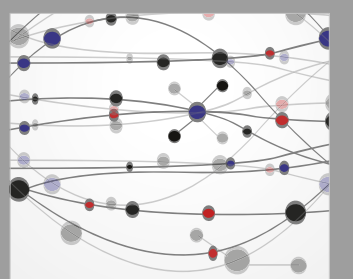

\section{Rotating \\ Machinery}

The Scientific World Journal

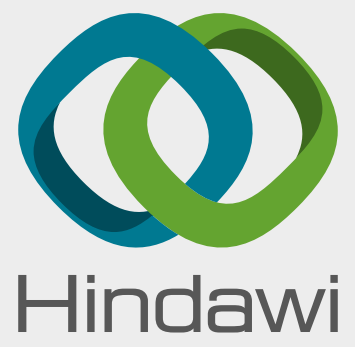

Submit your manuscripts at

www.hindawi.com
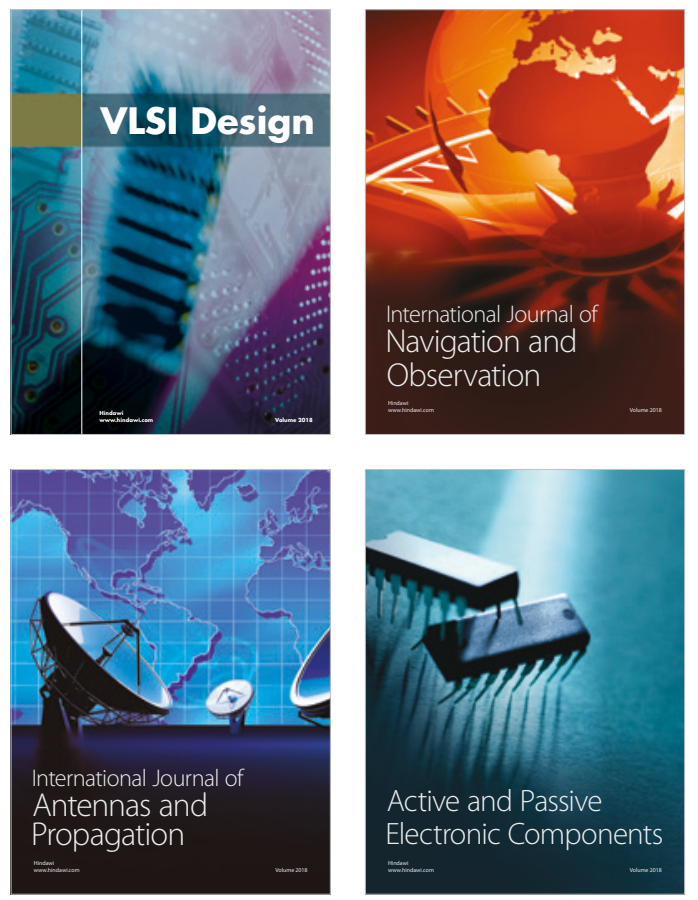
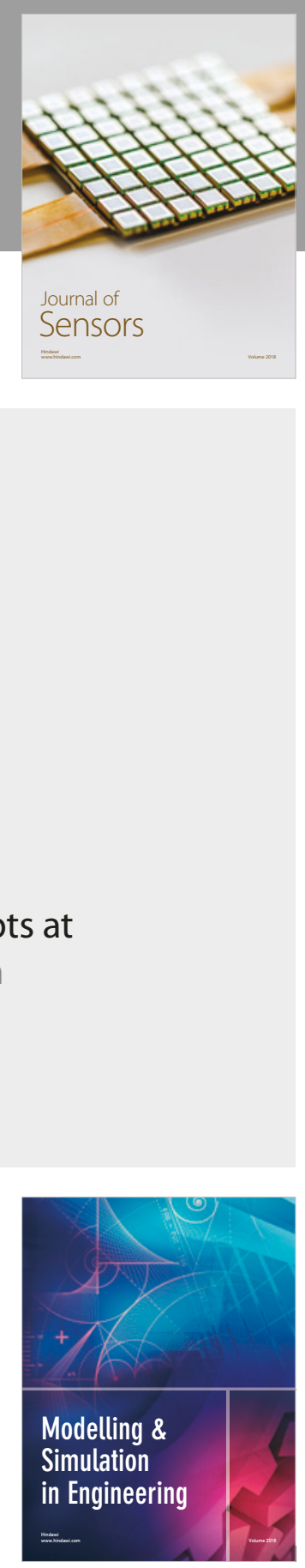

\section{Advances \\ Multimedia}
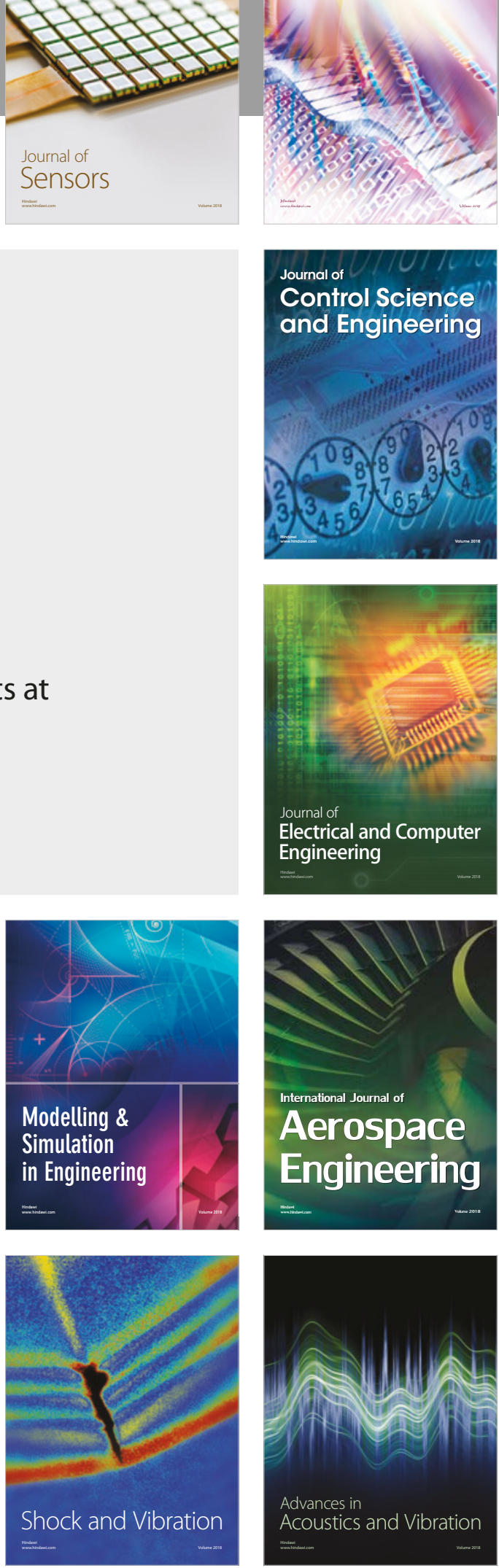